JOURNAL

of Health Inequalities

\title{
Eliminating racial and ethnic disparities in behavioral healthcare in the United States
}

\author{
Hoa Appel', Phuoc D. Nguyen², Kelly Bang ${ }^{2}$ \\ 'University of Washington Bothell, United States \\ 2Des Moines Medical College, United States
}

\begin{abstract}
This article discusses individual and systemic barriers to accessing behavioral healthcare, tailoring the examination to the specific needs of Asian Americans (AAs). Some barriers include language barriers, lack of access to behavioral health care, and providers that may not be culturally sensitive to patients' needs. These barriers lead to the underuse of health services and worse mental health outcomes. AAs are said to be the "model minority," a term most often to describe Chinese Americans, East Asians or Southeastern Asians. This is a myth; AAs with behavioral health needs encounter numerous barriers. Among the barriers are health insurance coverage, especially Medicaid expansion, which is not available in every state. Also, healthcare workers and providers are not always prepared to treat behavioral health needs in their primary care practice. This paper highlights the need to further investigate these topics in order to help eliminate the disparities that still exist in our growing populations of racial and ethnic minorities from the standpoint of healthcare practitioners in their practices.

Asian Americans now comprise the largest growing minority group in the United States (US). Asian Americans include all those that may be born in any Asian countries, or born in the US to parents who are not native English speakers. However, there remains insufficient comprehensive discussion and action to reduce the barriers to behavioral healthcare they face. It is important to tailor an all-inclusive agenda with the purpose of eliminating racial and ethnic disparities in behavioral healthcare for AAs in the US.
\end{abstract}

KEY WORDS: Asian American, model minority, health insurance, mental health, behavioral health, health disparity.

ADDRESS FOR CORRESPONDENCE: Hoa Appel, University of Washington Bothell, United States,

e-mail: happel@uw.edu

\section{INTRODUCTION}

Asian Americans (AAs), individuals of East, Southeast, and South Asian origins, are the fastest growing group in the United States (US), increasing from 11.9 million in 2000 to 22.3 million in 2019 [1]. Their rapid population growth means that AAs are projected to be the nation's largest immigrant group, surpassing Hispanic population growth by 2055 [2]. AAs are said to be the "model minority", a term associated with hardworking and overachieving Chinese Americans or East Asians, which overgeneralizes the overall AA population as more academically, economically, and socially successful than other minority groups $[3,4]$. AAs have been deemed as models for their success and their achieve- ments, attributed to their hard work and belief in the American meritocracy [5]. This view of AAs fails to take into account their historical and social circumstances. It is also an overgeneralization of the Asian ethnic group as a whole.

The model minority stereotype is a myth insofar as it inaccurately assumes all AAs are intrinsically talented and accomplished [5]. The myth discounts the racial disparities AAs have historically experienced and continue to face today. Internalizing the model minority myth can trigger chronic racism-related stress and potentially harm AAs mental and physical health in the long run [5]. Additionally, the pressure to live up to the model minority myth can put a strain in social relationships with family 
and friends. A recent study suggests social support is a protective factor for mental health disorders such as major depressive disorders and generalized anxiety disorder, among AAs [6]. In fact, AAs has a $17.3 \%$ overall lifetime rate of any psychiatric disorder in the US [7]. It is hypothesized that some of these factors may result in feeling burdened by unfair social pressure and the struggle to conform to meet society's unrealistic expectation as the model minority [3-5].

Behavioral health (BH) includes both mental health and substance use. Examples of $\mathrm{BH}$ concerns in AAs include drug and alcohol abuse $[8,9]$. The use of tobacco, alcohol, or illicit drugs may be an effort to treat depression or anxiety [4]. Furthermore, there is a greater prevalence of suicidal ideation among high school AAs compared to other races/ethnic groups [4]. Despite a greater risk of mental illness in this model minority group, studies show AAs are $44 \%$ less likely to have had a history of diagnosed depression and $40 \%$ less likely to have sought help for depression and/or anxiety $[4,10]$. These figures indicate a significant portion of AAs are not obtaining legitimate treatment for mental health needs.

Mental illness does not exist in isolation and often arises from interpersonal conflict and/or stress. In addition to the social pressure to meet unreasonable "model minority" expectations, other conditions that AAs report cause them mental illnesses include cultural adaptation and culturally-related parenting values and practices [11]. Specifically, parent-child conflicts within South Asian American families result in mental health challenges [11]. Taking into account acculturation and generational status, families struggle with biculturalism and find difficulty balancing values of Eastern and Western culture and parenting styles [11]. Additionally, mental illness can arise from external factors such as immigration issues. Refugees forced to flee their homelands may experience long-term psychological distress that affects their physical and mental well-being [12]. The resulting posttraumatic stress can persist years after resettling, have a negative impact on the current family dynamic, and later bring about intergenerational trauma [12].

When AAs seek professional mental health services, providers should be mindful of the influences a patient's social network has on his or her medical decision making [13]. An individual's social circle often helps him or her identify symptoms and seek mental health services. Therefore, friends, family members, and colleagues can either encourage or deter an individual from receiving necessary care [13]. In addition, one's social network may cause AAs to delay seeking health services $[11,13]$.

The literature search was conducted using PubMed and PsycINFO with search terms such as mental health, substance use, health disparity, inequity, model minority, challenges, and Asian American. The aim of this paper is to analyze the barriers to $\mathrm{BH}$ care faced by AAs in the US and discuss how to remove them. Our focus are to eliminate racial and ethnic health disparities and to highlight areas where $\mathrm{BH}$ can be improved to enhance patient health outcomes.

\section{BARRIERS TO MENTAL HEALTHCARE UNIVERSAL APPROACH}

Individual and systemic barriers stem from a universal approach to mental healthcare, where AA culture does not fit the norm. Examples of such barriers are AAs' cultural mistrust of the mental health system [14], the reluctance to admit mental health problems to others $[15,16]$, and language barriers [16]. These barriers can deter would-be patients from seeking psychological help [14]. Each ethnic subgroup has distinct beliefs and opinions on mental health that affect its propensity to seek healthcare [17]. Further heterogeneity in the Asian subgroup shows that Southeast Asians have a higher prevalence of psychiatric disorders (34.6\%) than East Asians (22.5\%) and South Asians (24.5\%) [18]. Filipino Americans were found to seek mental health services at a much lower rate than their other Asian counterparts [14]. Systemic barriers include a general lack of access to $\mathrm{BH}$ services. These barriers deserve a tailored approach to receiving care in order to address the mental health needs.

There are a few key reasons why AAs might not seek needed BH help. First, there is a lack of awareness of and concurrent difficulty recognizing the need for help during a mental health crisis $[10,15]$. Second, there are differences in cultural beliefs about health and helping. AAs are more likely to attribute $\mathrm{MH}$ issues to religious or other culturally-related beliefs which may lead to conflict, stigma, and shame, resulting in an unwillingness to disclose them to anyone $[8,15]$. Third, and related, AAs are less likely to report $\mathrm{MH}$ issues to peers or medical providers and to seek care due to fear of being labeled crazy, or reliving a previous poor experience following such a report $[10,15]$. Lastly, perceived discrimination and the model minority myth contribute to the decrease in mental health seeking [5, 8]. Only $17 \%$ of Asian Americans report any psychiatric disorder and only $9 \%$ seek help for any type of mental health services, compared with $18 \%$ for the general population nationwide [7].

Other barriers include language, avoidance of providers of a different ethnicity, perceived discrimination, and reluctance to remain in treatment. Limited English-speaking skills in AAs, including less exposure to services in their preferred language, set up a barrier to seeking mental health help [10]. Some Vietnamese Americans prefer to seek spiritual help [19], or non-professional help sources such as from family or friends [10]. This is unfortunate since young AA adults have higher rates of psychological distress than other groups [8]. Individuals with self-reported social stress and perceived discrimination are more likely to have depressive disorder and not seek treatment, for either mental illness or for 
substance use disorders [8]. Also, individuals who view their racial and ethnic minority group as less worthy are more likely to downgrade themselves accordingly, causing lower self-esteem in comparison to their peers $[8,20]$.

\section{WAYS TO ADDRESS BEHAVIORAL HEALTH DISPARITY}

Providing healthcare providers who speak the same language as their patients and an informal setting for them to meet can help alleviate mental health disparity. Vietnamese Americans who prefer non-professional help prefer education delivered in less formal, community-based settings [19]. Available resources should be in AA's native language to help them recognize their need for help and treatment [10]. Also, there should be more collaboration between formal service systems and community resources. A one-stop location would help. Moreover, mental health service agencies and providers should consider partnering with community organizations and faith-based communities [19].

Research shows that patients see their primary care providers for both physical and behavioral health needs. Therefore, $\mathrm{BH}$ care should be integrated in every healthcare system. An increasing number of general practitioners treat $\mathrm{BH}$ conditions but barriers such as language, access, insurance, and stigma conspire to discourage obtaining treatment [16]. Providers should know about the effects of stigma and shame in order to properly diagnose and help their AA patients. Providers must remember to dispel the model minority myth and the stigma and shame around mental illness $[4,21]$.
Given the heterogeneity in Asian subgroups, cultural and linguistic concordance might not accommodate every patient. For example, a person's ethnic minority group influences whether he or she views a problem as mental health related based on cultural norms [13]. Some cultures view their mental health problems as physical illnesses. Individuals from such an ethnic group will require a provider who is culturally competent to explain their condition and need for mental health services to them.

\section{ADDRESSING PREFERENCE OF DIVERSE POPULATIONS}

Research shows limited facets of diverse populations' preferences are being addressed. Behavioral health service delivery does not explain how minority patients prefer to be helped. Training providers to be culturally competent is a good start but the infrastructure that supports culturally competent providers must be improved [21]. Furthermore, the lack of cultural competency and implicit biases cause providers to underdiagnose this population with substance abuse disorders [21]. Also, AAs were perceived as having higher alcohol tolerance [9]. It is, therefore, imperative to train providers to remain as objective as possible. This is especially so where patient and provider are of different race or ethnicity, and for people with multiple chronic conditions and behavioral health comorbidities such as depression and anxiety, which lead to poor health outcomes $[16,22]$.

Effective provider-patient interaction requires some language understanding, familiarity with the patient's

\begin{tabular}{|c|c|c|}
\hline $\begin{array}{l}\text { Society barriers } \\
\text { - Lack of insurance coverage } \\
\text { - Availability of health services in minority } \\
\text { neighborhoods } \\
\text { - Interpretation services } \\
\text { - Racial match of providers }\end{array}$ & & $\begin{array}{l}\text { Society solutions } \\
\text { - Expand Medicaid for all states } \\
\text { - Increase community health centers } \\
\text { - Full coverage for Interpretation services } \\
\text { - Recruit and expand diversity of providers }\end{array}$ \\
\hline $\begin{array}{l}\text { Interpersonal barriers } \\
\text { - Language compatibility between patient } \\
\text { and provider } \\
\text { - Preconceived discrimination } \\
\text { and prejudice } \\
\text { - Cultural heterogeneity }\end{array}$ & $\begin{array}{l}\text { Health } \\
\text { disparity }\end{array}$ & $\begin{array}{l}\text { Interpersonal solutions } \\
\text { - Cultural competency training for providers } \\
\text { - Use of language technology and/or third } \\
\text { party } \\
\text { - Continuity of care with PCP }\end{array}$ \\
\hline $\begin{array}{l}\text { Intrapersonal barriers } \\
\text { - Economic repercussions } \\
\text { - Embarrassment/shame } \\
\text { - Cultural beliefs } \\
\text { - Belief that mental illness is caused } \\
\text { by external factors }\end{array}$ & & $\begin{array}{l}\text { Intrapersonal solutions } \\
\text { - Education on confidentiality of health } \\
\text { information } \\
\text { - Increased information on mental health } \\
\text { illness } \\
\text { - Awareness of mental health } \\
\text { and de-stigmatization }\end{array}$ \\
\hline
\end{tabular}

FIGURE 1. Factors contributing to health disparity 
cultural preferences, with unbiased and easy to understand explanations $[23,24]$. Cultural factors, such as the language around mental health, ethnic identity, and a holistic approach to the mind and body predicts an increased use of mental health services in this patient population [24]. Help-seeking behaviors vary according to nativity, language proficiency, and cultural conflict [23]. Patients with higher English proficiency who were born in the USA are more likely to seek care [23]. Asian Americans are also more likely to consult family members before making medical decisions, an important factor to consider when delivering care $[11,24]$.

The stigma associated with using mental health services can be so great that patients will often turn to their close social network first, such as friends and family before seeking professional help [25]. The stigma is so pervasive that Asian Americans still do not seek medical help even when available $[24,25]$. In a study regarding opinions of depression held by Asian Americans, 37.1\% perceived it as a sign of weakness in addition to bringing shame to the family [17]. Family members were shown to actively discourage professional help-seeking to "save face" [23].

\section{INSURANCE COVERAGE}

Even though more uninsured patients receive health coverage under the Affordable Care Act (ACA) in 2010, minorities remain less likely to be insured and continue to lack healthcare access. While the ACA allowed Medicaid and marketplace expansion in 2014, the Asian American uninsured rate remains higher than the rate for whites [26]. Even though the Medicaid waiver allows more insurance coverage for low-income recipients, particularly those in the rural areas, about 13 states lack the Medicaid waiver [27]. Minority groups, the uninsured, low-income, and people living in minority neighborhoods tend to experience lower access to $\mathrm{BH}$ compared with the general population [28]. Even those with insurance may encounter specific barriers to receiving care depending on insurance type. Those on state sponsored Medicaid see their insurance rejected by primary care offices more frequently than did those on employer sponsored coverage (10.93\% vs. 3.31\%) [29]. The access to specialist care gap is even more pronounced with $21.62 \%$ of Medicaid-only insurance being rejected compared to $7.87 \%$ of employer-sponsored coverage [29].

\section{DIVERSIFYING AND EXPANDING BEHAVIORAL HEALTH WORKFORCE}

Asian Americans are more likely to use primary care than specific mental health services [24]. The World Health Organization recognizes that integration of behavioral health into primary care will increase the use of health services and improve health outcomes [30]. Health outcomes improve with this integration: patients have a first point of contact with their primary care phy- sician in addition to having lifetime relationships with their providers [31]. Integrating primary care with $\mathrm{BH}$ care allows patients to address their mental health and substance use issues with one visit [31].

Primary care provides continuity of care to patients for improved patient outcomes. Patients with mental health disorders who visit their psychiatrist at least twice over six months live longer than their counterparts with lower rates of continuity of care [32]. Furthermore, patients with high continuity of care from their primary care provider (PCP) are significantly less likely to be hospitalized and with lower health care costs [33]. These factors predict success for patients in reducing disparities and costs and relief for the strained healthcare system. With the increasing role of PCPs treating those with $\mathrm{BH}$ concerns, primary care is poised to integrate $\mathrm{BH}$ services with primary care [34].

\section{CONCLUSIONS}

Though AAs are the fastest growing ethnic minority group in the US, there is scant research in public health regarding barriers to behavioral health in relation to $\mathrm{AA}^{\prime}$ subgroups. Research is limited as there are over thirty ethnic groups classified as "Asian" and none is representative of the whole. Heterogeneity among AAs should be considered when diagnosing mental health illness and how to best increase their access to healthcare. For example, Vietnamese Americans are more underinsured compared to Filipino and Chinese Asian Americans, making it harder for them to seek care [35].

More Asian healthcare providers are needed in order to reduce individual and systemic barriers. Individualized care with sensitivity and responsiveness to cultural heterogeneity must be employed in order to address adequately the individual needs of each patient. Community clinics with culturally competent care are needed to make patients more comfortable seeking care. Since primary care providers see many types of patients, this highlights the need to provide behavioral health services in primary care.

\section{DISCLOSURE}

The authors report no conflict of interest.

\section{References}

1. Agency for Healthcare Research and Quality. National Healthcare Quality and Disparities Report. Available from: https:// archive.ahrq.gov/research/findings/nhqrdr/nhqdr14/2014nhqdr.pdf (accessed: 20.02.2021).

2. Key facts about Asian Americans, a diverse and growing population. Available from: https://www.pewresearch.org/facttank/2017/09/08/key-facts-about-asian-americans/ (accessed: 21.01.2021).

3. Kiang L, Witkow MR, Thompson TL. Model minority stereotyping, perceived discrimination, and adjustment among ado- 
lescents from Asian American backgrounds. J Youth Adolesc 2016; 45(7): 1366-1379.

4. Sabato T. Breaking the model minority stereotype: an exploration of Asian American and Pacific Islander student substance abuse. Coll Student J 2016; 50: 477-488.

5. Yoo HC, Miller MJ, Yip P. Validation of the internalization of the Model Minority Myth Measure (IM-4) and its link to academic performance and psychological adjustment among Asian American adolescents. Cult Diver Ethnic Min Psychol 2015; 21(2): 237-246.

6. Sangalang CC, Gee GC. Depression and anxiety among Asian Americans: the effects of social support and strain. Soc Work 2012; 57(1): 49-60.

7. Spencer MS, Chen J, Gee GC, et al. Discrimination and mental health-related service use in a national study of Asian Americans. Am J Pub Health 2010; 100(12): 2410-2417.

8. Chae $\mathrm{DH}$, Lee $\mathrm{S}$, Lincoln $\mathrm{KD}$, et al. Discrimination, family relationships, and major depression among Asian Americans. J Immig Min Health 2011; 14(3): 361-370.

9. Cheng AW, Lee CS, Iwamoto DK. Heavy drinking, poor mental health, and substance use among Asian Americans in the NLAAS: a gender-based comparison. PsycEXTRA Dataset, 2011.

10. Chu JP, Hsieh KY, Tokars DA. Help-seeking tendencies in Asian Americans with suicidal ideation and attempts. Asian Am Journal Psychol 2011; 2(1): 25-38.

11. Sharma N, Shaligram D, Yoon GH. Engaging South Asian youth and families: A clinical review. Int J Social Psych 2020; 66(6): 584-592.

12. Sangalang CC, Vang C. Intergenerational trauma in refugee families: A systematic review. J Immig Min Health 2016; 19(3): 745-754.

13. Nguyen D, Bornheimer LA. Mental health service use types among Asian Americans with a psychiatric disorder: considerations of culture and need. J Behav Health Serv Res 2014; 41(4): 520-528.

14. David EJ. Cultural mistrust and mental health help-seeking attitudes among Filipino Americans. Asian Am J Psychol 2010 1(1): 57-66

15. Shi W, Shen Z, Wang S, et al. Barriers to professional mental health help-seeking among Chinese adults: A systematic review. Fron Psych 2020; 11: 442.

16. Weng SS, Spaulding-Givens J. Strategies for working with Asian Americans in mental health: community members' policy perspectives and recommendations. Admin Pol Ment Health Ment Health Serv Res 2017; 44(5): 771-781.

17. Jung H, Cho YJ, Rhee MK, et al. Stigmatizing beliefs about depression in diverse ethnic groups of Asian Americans. Comm Ment Health J 2019; 56(1): 79-87.

18. Lee SY, Martins SS, Lee HB. Mental disorders and mental health service use across Asian American subethnic groups in the United States. Comm Ment Health J 2014; 51(2): 153-160.

19. Kim-Mozeleski JE, Tsoh JY, Gildengorin G, et al. Preferences for depression help-seeking among Vietnamese American adults. Comm Ment Health J 2017; 54(6): 748-756.

20. Augsberger A, Yeung A, Dougher M, et al. Factors influencing the underutilization of mental health services among Asian
American women with a history of depression and suicide. BMC Health Serv Res 2015; 15(1): 542.

21. Cheng AW, Iwamoto DK, McMullen D. Model minority stereotype and the diagnosis of alcohol use disorders: Implications for practitioners working with Asian Americans. J Ethn Sub Abuse 2016; 17(3): 255-272.

22. Huang B, Appel HB, Nicdao EG, et al. Chronic conditions, behavioral health, and use of health services among Asian American Men. Am J Mens Health 2013; 7(1): 66-76.

23. Kang SY, Howard D, Kim J, et al. English language proficiency and lifetime mental health service utilization in a national representative sample of Asian Americans in the USA. J Pub Health 2010; 32(3): 431-439.

24. Kwok J. Factors that influence the diagnoses of Asian Americans in mental health: an exploration. Pers Psychia Care 2013; 49(4): 288-292.

25. Leong FT, Kim HH, Gupta A. Attitudes toward professional counseling among Asian-American college students: acculturation, conceptions of mental illness, and loss of face. Asian Am J Psych 2011; 2(2): 140-153.

26. Park JJ, Humble S, Sommers BD, et al. Health insurance for Asian Americans, Native Hawaiians, and Pacific Islanders under the Affordable Care Act. JAMA Int Med 2018; 178(8): 1128.

27. Status of State Medicaid expansion decisions: Interactive map. Available from: https://www.kff.org/medicaid/issue-brief/status-of-state-medicaid-expansion-decisions-interactive-map/ (accessed: 21.01.2021).

28. Yang KG, Rodgers CR, Lee E, et al. Disparities in mental health care utilization and perceived need among Asian Americans: 2012-2016. Psych Serv 2020; 71(1): 21-27.

29. Alcalá HE, Roby DH, Grande DT, et al. Insurance type and access to health care providers and appointments under the Affordable Care Act. Med Care 2018; 56(2): 186-192.

30. Integrating mental health into primary care: a global perspective. Available from: http://www.who.int/mental_health/resources/ mentalhealth_PHC_2008.pdf (accessed: 21.01.2021).

31. Funk M, Saraceno B, Drew N, et al. Integrating mental health into primary healthcare. Ment Health Fam Med 2008; 5(1): 5-8.

32. Bazemore A, Petterson S, Peterson LE, et al. Higher primary care physician continuity is associated with lower costs and hospitalizations. Ann Fam Med 2018; 16(6): 492-497.

33. Chen J, Vargas-Bustamante A, Mortensen K, et al. Racial and ethnic disparities in health care access and utilization under the Affordable Care Act. Med Care 2016; 54(2): 140-146.

34. Olfson $\mathrm{M}$. The rise of primary care physicians in the provision of US mental health care. J Health Polit Policy Law 2016; 41(4): 559-583.

35. Alegría M, Cao Z, McGuire TG, et al. Health insurance coverage for vulnerable populations: contrasting Asian Americans and Latinos in the United States. Inquiry 2006; 43(3): 231-254.

\section{AUTHORS' CONTRIBUTIONS}

HA prepared research concept and design of the publication and analysed data. All authors collected data, wrote the article, contributed to preparing the final version of publication. HA, PDN critically revised it. 